\title{
Finite-basis many-electron approximation to the Anderson model
}

\author{
Jay D. Mancini \\ Department of Physics, Virginia Tech, Blacksburg, Virginia 24061* \\ Samuel P. Bowen \\ Department of Physics, Fordham University, The Bronx, New York 10458 \\ Yu Zhou \\ Department of Physics, Virginia Tech, Blacksburg, Virginia 24061 \\ (Received 4 August 1988; revised manuscript received 28 August 1989)
}

\begin{abstract}
A relatively simple many-electron basis is used to construct a matrix for the Anderson impurity Hamiltonian. The basis states are each valid in the thermodynamic limit. The approximate ground-state energy compares well with Bethe-ansatz results for large Coulomb energies. The ground-state wave-function properties are not as well approximated. This method may be well suited to studies of more realistic Hamiltonians and their ground-state energy and its derivatives.
\end{abstract}

This paper presents a variational matrix method for calculating the ground-state energy of Anderson-like models. This method is modest in its analytic and numerical requirements and applicable to more complicated Hamiltonians. The method is an extension of some preliminary earlier work. ${ }^{1}$ Here we will compare with the ground-state properties of the impurity Anderson model ${ }^{2}$ as evaluated by Kawakami and Okiji (KO). ${ }^{3}$

The variational many-particle basis states correspond to averages of the individual elementary excitations and deviations about these averages. These states are closely related to the moments of the excitation energies of the elementary excitations. In this paper a simple approximation with an $18 \times 18$ matrix gives, in some parameter ranges, reasonably good results with very little effort.

This technique generates a sequence of many-electron states from the filled Fermi sea by repeated operations with the Hamiltonian to create orthonormal states, each of which has a proper thermodynamic limit, and represents a simple physical process. The major difference between this approach and others ${ }^{4}$ is that the number of such vectors is kept quite small and the matrices that have to be analyzed are also small. ${ }^{5}$ In the following we use the well-known Anderson model Hamiltonian

$$
\begin{aligned}
H= & \sum_{k, s} \varepsilon_{k s} n_{k s}+\sum_{k, s} V(k)\left(c_{k s}^{\dagger} f_{s}+f_{s}^{\dagger} c_{k s}\right) \\
& +\sum_{s} E_{s} N_{s}+U N_{\uparrow} N_{\downarrow}
\end{aligned}
$$

where $E_{s}$ is the atomic orbital energy of the localized orbitals, $U$ is the Coulomb repulsion integral, and $V(k)$ is the hybridization integral for the mixing of the localized orbitals $f_{s}$ and the conduction electrons $c_{k s}$ whose band energies are $\varepsilon_{k s}$. In the work that follows we assume that $V(k)=V$ is independent of $k$. We chose our initial state $|0\rangle$ to be the filled Fermi sea and the empty impurity orbital.
The first row of the Hamiltonian secular matrix is

$$
H|0\rangle=E_{0}|0\rangle+N^{-1 / 2} V \sum_{k, s} f_{s}^{\dagger} c_{k s}|0\rangle .
$$

Since the impurity is empty, terms involving $U$ and $c_{k s}^{\dagger} f_{s}$ are absent. We start the orthogonal sequence with the second term in Eq. (2). These terms contain all of the localized-particle conduction electron-hole excitations. This first vector in our discrete basis is the sum of the first set of vectors in the variational papers. ${ }^{6}$ In contrast we make each spin index a different state and require further states in the basis to represent distinct physical processes rather than maintain a tridiagonal matrix. The norms squared of these first vectors $\left|\Psi_{1 s}\right\rangle$ are given by

$$
x_{s}=N^{-1} \sum_{k}\left\langle n_{k s}\right\rangle \text {, }
$$

where $\left\langle n_{k s}\right\rangle$ is the Fermi-function occupation in the noninteracting Fermi state $|0\rangle$. The action of $H$ on these two first vectors is

$$
\begin{aligned}
H\left|\hat{\Psi}_{1 s}\right\rangle= & \left(E_{0}+E_{f}-\bar{\varepsilon}_{s}^{<}\right)\left|\hat{\Psi}_{1 s}\right\rangle \\
& +x_{s}^{1 / 2} V|0\rangle-\frac{\sigma_{s}}{x_{s}^{1 / 2}}\left|\hat{\psi}_{1 \delta s}\right\rangle,
\end{aligned}
$$

where the normalization $\sigma_{s}$ is defined in Appendix A. The first of the new vectors in Eq. (4) illustrates the first-excitation-energy-moment state in the basis. This vector is defined by

$$
\left|\psi_{1 \delta s}\right\rangle=N^{-1 / 2} \sum_{k}\left(\varepsilon_{k s}-\bar{\varepsilon}_{s}^{<}\right) f_{s}^{\dagger} c_{k s}|0\rangle,
$$

where the average conduction-electron energy is chosen as

$$
\bar{\varepsilon}_{s}^{<}=\frac{1}{x_{s}} \frac{1}{N} \sum_{k} \varepsilon_{k s} n_{k s}
$$


so that $\left\langle\hat{\Psi}_{1 s} \mid \hat{\Psi}_{1 \delta}\right\rangle=0$. As the further vectors are generated by higher powers of $H$, a sequence of vectors of the form

$$
\left|\phi_{n}\right\rangle=\frac{1}{\sqrt{N}} \sum_{k}\left(\varepsilon_{k s}-\bar{\varepsilon}_{s}^{<}\right)^{n} f_{s}^{\dagger} c_{k s}|0\rangle
$$

will be generated. The other states in Eq. (4) are also of the moment type for other physically distinct excitations. The first type are zeroth-moment conduction-electron particle-hole excitations

$$
\left|\Psi_{p h s}\right\rangle=\frac{1}{\sqrt{N}} \sum_{k, k^{\prime}} c_{k s}^{\dagger} c_{k^{\prime} s}|0\rangle,
$$

and the double-occupancy $f$-state vector is

$$
\left|\Psi_{2 f}\right\rangle=\frac{1}{N} \sum_{k, k^{\prime}} f_{\uparrow}^{\dagger} f_{\downarrow}^{\dagger} c_{k \downarrow} c_{k^{\prime} \uparrow}|0\rangle .
$$

The vectors for the 18-dimensional basis which is used for comparison with the Bethe-ansatz (BA) results in this paper are given in Appendix A. The matrix of the Hamiltonian in this basis is quite sparse and is presented in Appendix B. The matrix elements are functions of averages of polynomials of excitation energies over the filled noninteracting Fermi sea.

The ground-state energy is displayed in Fig. 1. The break in the solid line indicates the original small- $U$ values of $\mathrm{KO}$ (Ref. 3) and a calculation of their results for large- $U$ values recalculated for this paper. The agreement between the matrix lowest energy and the Bethe Anzatz is not good for small $U$ where perturbation theory and Hartree-Fock are good approximations. However, the agreement becomes quite good as $U$ increases.

The magnetic moment as a function of magnetic field is shown in Fig. 2. The truncation approximation is partic-

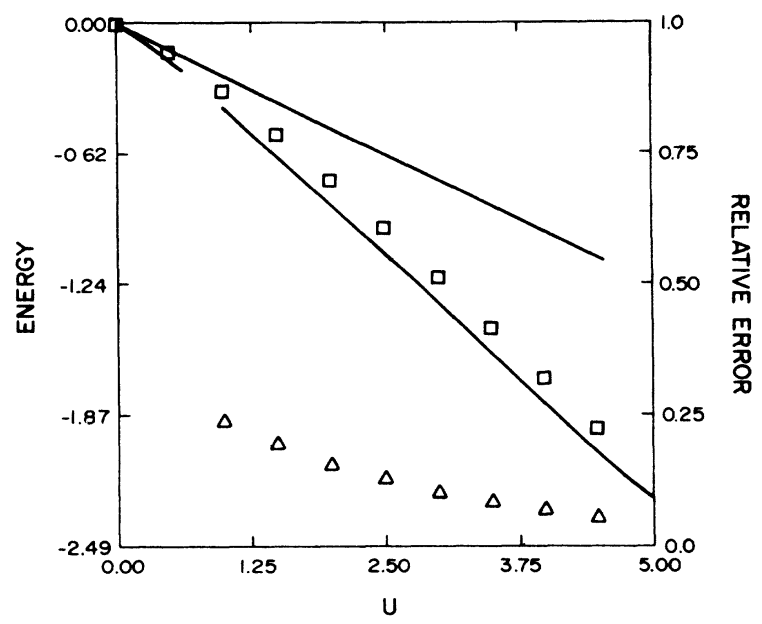

FIG. 1. The variational ground-state energy is compared to the exact Bethe-anzatz (BA) results. The squares are the ground state of the $18 \times 18$ matrix truncation. The lower solid curve is the exact BA results. The higher solid curve is the HartreeFock approximation and the triangles are the relative error (right axis). The small exact $U$ values are from Ref. 3 and the large- $U$ values are an extension of the exact BA energy.

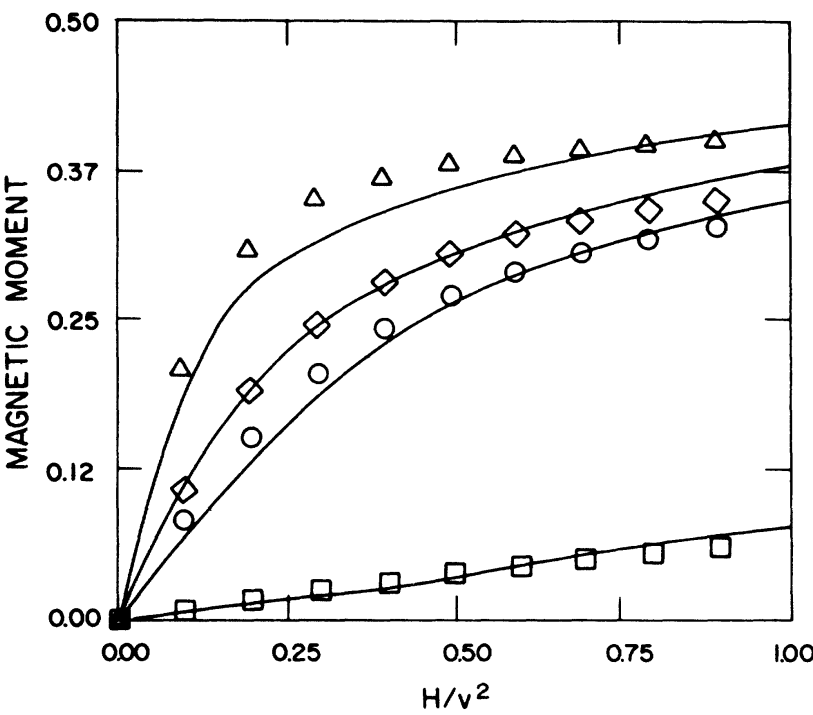

FIG. 2. The magnetic moment of the impurity electron for the Anderson model calculated in the $18 \times 18$ truncation and compared to the BA results of Ref. 4 for various value of $U / V^{2}$, $U / V^{2}=0(\square), U / V^{2}=1.5(\bigcirc), U / V^{2}=2.0(\diamond), U / V^{2}=15$ $(\triangle)$.

ularly good for small and large $U$ for the symmetric model. However, for intermediate $U$ values the magnetic field dependence is more abrupt than the exact results. This is a manifestation of the limited number of vectors included in the truncation approximation. The magnetic susceptibility is compared to the BA results in Fig. 3. Here we compare the dependence of the susceptibility as a function of the localized orbitals $\varepsilon_{f}$. In the mixed valence region we find very close agreement, but the peak is slightly displaced from the exact results. In the Kondo regime the values of the susceptibility are again close. The

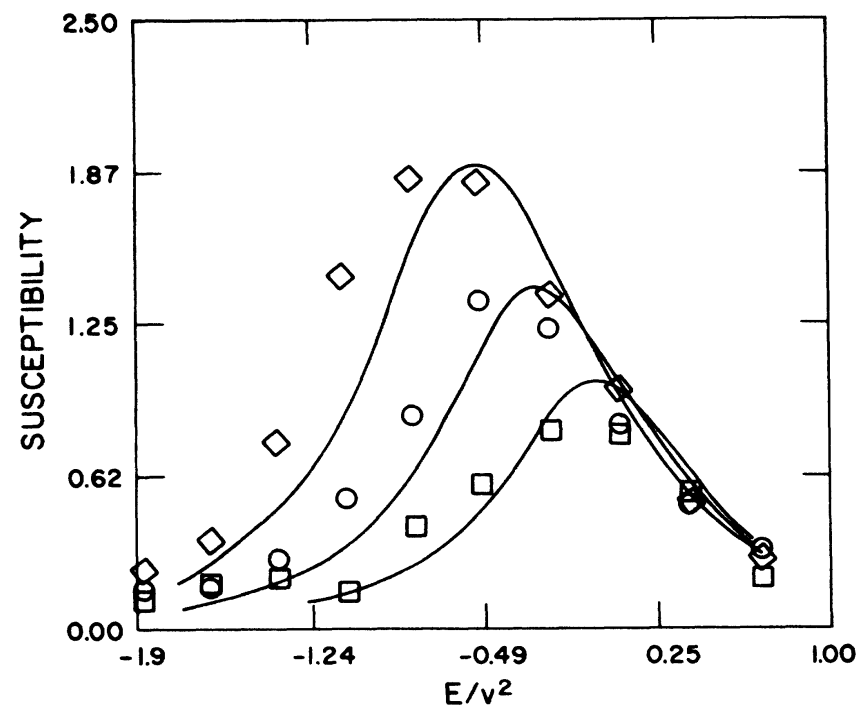

FIG. 3. The impurity susceptibility as a function of the local electron orbital energy for differing values of $U$. The solid lines are the BA results of Ref. 4 and the curves correspond to $U / V^{2}=0.0$ (squares), $U / V^{2}=0.5(\bigcirc)$, and $U / V^{2}=1.0(\diamond)$. 


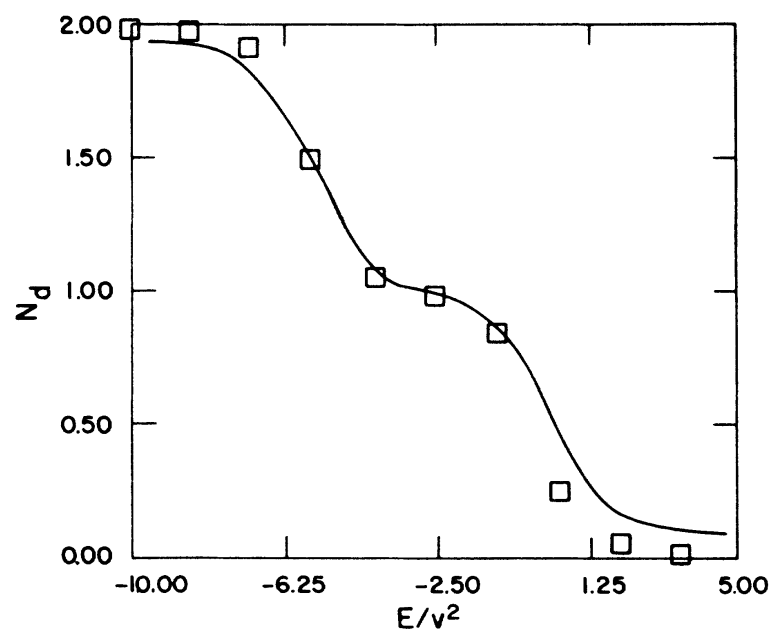

FIG. 4. The ground-state occupation of the impurity electron orbital as a function of the local electron orbital energy $E$. The solid line represents the exact BA results of Ref. 4 and the squares represent the $18 \times 18$ truncation.

dependence of the occupation of the localized orbitals $n_{f}$ is shown in Fig. 4. Because of the finite basis size of the truncation $n_{f}$ also approaches the limits of 0 and 2 more quickly than the exact results. All of these comparisons are made with the same set of model parameters.

The charge susceptibility $\chi_{c}$ was also studied for this truncation. The dependence of $\chi_{c}$ on $\varepsilon_{f}$ and $U$ is qualitatively correct, achieving the correct limiting behavior as the $f$ level approaches or is far from the Fermi energy. However, rate of change of $\chi_{c}$ with model parameters is significantly slower than the exact calculations. Using $\chi_{c}$ and $\chi_{s}$ to calculate the Wilson ratio with this method results in correct limiting behaviors, but only a semiquantitative agreement in between.

If a greater effort had been made to generate a larger matrix, it is expected that the results would be closer. The relative success of these moment-generated manyelectron basis states suggests that they might be useful for the two-impurity or full-lattice model.

\section{APPENDIX A: THE BASIS VECTORS USED IN THE TRUNCATION}

The basis vectors used in the truncation approximation are included in the listing below. A caret over a vector indicates it is a unit vector. The vector with smaller index corresponds to spin up. Vector 1 is the Fermi sea and the empty localized state. Vectors 2 and 3 are the mixed particle-hole excitations defined in Eqs. (2) and (3). Vectors 4 and 5 are the first moments of the mixed particle-hole excitations, were defined in Eq. (5), and have a norm squared of

$$
\sigma_{s}^{2}=\frac{1}{N} \sum_{k}\left(\varepsilon_{k s}-\varepsilon_{s}^{<}\right)^{2} n_{k s} .
$$

Vectors 6 and 7 are conduction-electron particle-hole excitations and were defined in Eq. (8) and have a norm squared of $x_{s}\left(1-x_{s}\right)$. Vector 8 is the two- $f$ electron-two-conduction-hole state defined in Eq. (9) and whose norm squared is $x_{\uparrow} x_{\downarrow}$.

Vectors 9 and 10 are the first moment of the conduction-electron particle-hole excitations:

$$
\left|\Psi_{p h \delta s}\right\rangle=\frac{1}{\sigma_{s}^{<}} \frac{1}{N} \sum_{\substack{k^{\prime}>k_{f}, k<k_{f}}} \frac{\left(\varepsilon_{k s}-\varepsilon_{s}^{<}\right) c_{k^{\prime} s}^{\dagger} c_{k s}|0\rangle}{\left(1-x_{s}\right)^{1 / 2}} .
$$

Vectors 11 and 12 are first moments of the two- $f$ particle-two-conduction-hole excitations of vector 8 , and whose norm squared is $x_{s} \sigma_{s}^{<}$.

Vector 11: $\left|\Psi_{2 f \delta_{\uparrow}^{<}}\right\rangle$

$$
=\frac{1}{N} \sum_{k, k^{\prime}<k_{f}}\left(\varepsilon_{k \uparrow}-\varepsilon_{\uparrow}^{<}\right) f_{\uparrow}^{\dagger} f_{\downarrow}^{\dagger} c_{k^{\prime} \downarrow} c_{k \uparrow}|0\rangle,
$$

Vector 12: $\left|\psi_{2 f \delta^{<}}\right\rangle$

$$
=\frac{1}{N} \sum_{k, k^{\prime}<k_{f}}\left(\varepsilon_{k_{\downarrow}^{\prime}}-\varepsilon_{\downarrow}^{<}\right) f_{\uparrow}^{\dagger} f_{\uparrow}^{\dagger} c_{k^{\prime} \downarrow} c_{k \uparrow}|0\rangle \text {. }
$$

Vectors 13 and 14 are Gramm-Schmidt-orthonormalized vectors of the second-moment vector for localized-conduction particle-hole excitations:

$$
\left|\psi_{1 \delta_{s}^{2}}\right\rangle=\left|\phi_{1 \delta_{s}^{2}}\right\rangle-\frac{\left\langle\delta \varepsilon_{s}^{<2}\right\rangle}{\left(x_{s}\right)^{1 / 2}}\left|\hat{\psi}_{1 s}\right\rangle-\frac{\left\langle\delta \varepsilon_{s}^{<2}\right\rangle^{2}}{\sigma_{s}}\left|\psi_{1 \delta s}\right\rangle
$$

where

$$
\begin{aligned}
& \left\langle\delta \varepsilon_{s}^{<n}\right\rangle=\frac{1}{N} \sum_{k}\left(\varepsilon_{k s}-\varepsilon_{s}^{<}\right)^{n} n_{k s}, \\
& \left|\phi_{1 \delta_{s}^{2}}\right\rangle=\frac{1}{\sqrt{N}} \sum_{k}\left(\varepsilon_{k s}-\varepsilon_{s}^{<}\right)^{2} f_{s}^{\dagger} c_{k s}|0\rangle,
\end{aligned}
$$

and

$$
\left\langle\psi_{1 \delta_{s}^{2}} \mid \psi_{1 \delta_{s}^{2}}\right\rangle=\left\langle\delta \varepsilon_{s}^{4}\right\rangle-\frac{\left\langle\delta \varepsilon_{s}^{2}\right\rangle^{2}}{x_{s}}-\frac{\left\langle\delta \varepsilon_{s}^{3}\right\rangle^{2}}{\sigma_{s}^{2}}
$$

Vectors 15 and 16 are the first moment of the conduction-electron particle-hole excitations:

$$
\left|\psi_{p h \delta_{s}^{>}}\right\rangle=\frac{1}{N} \sum_{k^{\prime}>k_{F}} \sum_{k<k_{F}}\left(\varepsilon_{k^{\prime} s}-\varepsilon_{s}^{>}\right) c_{k^{\prime} s}^{\dagger} c_{k s}|0\rangle,
$$

where

$$
\varepsilon_{s}^{>}=\frac{1}{\sqrt{1-x_{s}}} \frac{1}{N} \sum_{k} \varepsilon_{k s}\left(1-n_{k s}\right)
$$

and

$$
\sigma_{s}^{>2}=\frac{1}{N} \sum_{k}\left(\varepsilon_{k s}-\varepsilon_{s}^{>}\right)^{2}\left(1-n_{k s}\right) .
$$

The 17 th and 18 th vectors are the first two-particle, two- 
hole state of mixed type whose norm squared is $\left(1-x_{s}\right) x_{s} x_{-s}$ :

$$
\left|\psi_{f s p h s}\right\rangle=\frac{1}{N^{3 / 2}} \sum_{k^{\prime}>k_{F}} \sum_{k<k_{F}} f_{\bar{s}}^{\dagger} c_{k^{\prime} s}^{\dagger} c_{k s} c_{k \bar{s}}|0\rangle .
$$

\section{APPENDIX B: THE HAMILTONIAN MATRIX}

The nonzero matrix elements of the Hamiltonian above and on the diagonal are listed below. Here $E_{0}$ is the energy of the filled Fermi sea. Where two elements are equal to an expression containing the spin index $s$ the smaller indices go with spin up and the larger with spin down.

$$
\begin{aligned}
& H_{11}=E_{0}, \\
& H_{12}=H_{13}=\sqrt{x_{s}} V, \\
& H_{22}=H_{33}=E_{0}+E_{s}-\varepsilon_{s}^{<}, \\
& H_{24}=H_{35}=-\sigma_{s}^{<} / \sqrt{x_{s}}, \quad H_{26}=H_{28}=\left(1-x_{s}\right)^{1 / 2} V, \\
& H_{37}=\sqrt{1-x_{\downarrow}} V, \quad H_{38}=\sqrt{x_{\uparrow}} V, \\
& H_{44}=H_{55}=E_{0}+E_{s}-\varepsilon_{s}^{<}-\left\langle\delta \varepsilon_{s}^{<3}\right\rangle / \sigma_{s}^{<2}, \\
& H_{4,13}=H_{5,14}=\frac{-1}{\sigma_{s}}\left[\left\langle\delta \varepsilon_{s}^{<4}\right\rangle-\frac{\left\langle\delta \varepsilon_{s}^{<2}\right\rangle^{2}}{x_{s}}-\frac{\left\langle\delta \varepsilon_{s}^{<2}\right\rangle^{3}}{\sigma_{s}^{2}}\right]^{1 / 2}, \\
& H_{4,9}=H_{5,10}=V, \\
& H_{4,11}=H_{5,12}=\sqrt{x_{s} V,} \\
& H_{6,6}=H_{7,7}=E_{0}+\varepsilon_{s}^{>}-\varepsilon_{s}^{<},
\end{aligned}
$$

$$
\begin{aligned}
& H_{6,15}=H_{7,16}=\sigma_{s}^{>} /\left(1-x_{s}\right)^{1 / 2} \text {, } \\
& H_{6,9}=H_{7,10}=-\sigma_{s}^{<} / \sqrt{x_{s}} \text {, } \\
& H_{6,17}=H_{7,18}=\sqrt{x_{s}} V \text {, } \\
& H_{8,8}=E_{0}+E_{\uparrow}+E_{\downarrow}+U-\varepsilon_{\downarrow}^{<}-\varepsilon_{\uparrow}^{<}, \\
& H_{8,11}=H_{8,12}=-\sigma_{s}^{<} / \sqrt{x_{s}} \text {, } \\
& H_{8,17}=H_{8,18}=\left(1-x_{s}\right)^{1 / 2} V \text {, } \\
& H_{9,9}=H_{10,10}=E_{0}+\varepsilon_{s}^{>}+\varepsilon_{s}^{<}-\left\langle\delta \varepsilon_{s}^{<3}\right\rangle / \sigma_{s}, \\
& H_{11,11}=H_{12,12} \\
& =E_{0}+E_{\uparrow}+E_{\downarrow}+U-\varepsilon_{\uparrow}^{<}-\varepsilon_{\downarrow}^{<}-\left\langle\delta \varepsilon_{s}^{<3}\right\rangle / \sigma_{s}, \\
& H_{13,13}=H_{14,14}=E_{0}+E_{s}-\varepsilon_{s}^{<}+A_{s} / B_{s} \text {, }
\end{aligned}
$$

where

$$
\begin{aligned}
& A_{s}=\left\langle\delta \varepsilon_{s}^{<5}\right\rangle-2\left\langle\delta \varepsilon_{s}^{<3}\right\rangle\left\langle\delta \varepsilon_{s}^{<4}\right\rangle / \sigma_{s} \\
&-2\left\langle\delta \varepsilon_{s}^{<2}\right\rangle\left\langle\delta \varepsilon_{s}^{<3}\right\rangle / \sqrt{x_{s}} \\
&+2\left\langle\delta \varepsilon_{s}^{<3}\right\rangle\left\langle\delta \varepsilon_{s}^{<2}\right\rangle^{2} / \sigma_{s} \sqrt{x_{s}} \\
&+\left\langle\delta \varepsilon_{s}^{<3}\right\rangle^{3} / \sigma_{s}^{2}, \\
& B_{s}=\left\langle\delta \varepsilon_{s}^{<4}\right\rangle-\left\langle\delta \varepsilon_{s}^{<2}\right\rangle^{2} / \sqrt{x_{s}}-\left\langle\delta \varepsilon_{s}^{<3}\right\rangle^{2} / \sigma_{s}, \\
& H_{15,15}=H_{16,16}=E_{0}+\varepsilon_{s}^{>}-\varepsilon_{s}^{<}+\frac{1}{\sigma_{s}^{>2}} \frac{1}{x_{s}}\left\langle\delta \varepsilon_{s}^{>3}\right\rangle, \\
& H_{17,17}=H_{18,18}=E_{0}+E_{-s}+\varepsilon_{s}^{>}-\varepsilon_{s}^{<}-\varepsilon_{-s}^{<} .
\end{aligned}
$$

${ }^{*}$ Present address: Division of Educational Programs, Argonne National Laboratory, Argonne, Illinois 60439.

1J. D. Mancini, C. D. Potter, and S. P. Bowen, J. Appl. Phys. 61, 3168 (1987); J. D. Mancini and D. C. Mattis, Phys. Rev. B 31, 7440 (1985); 29, 6988 (1984); 28, 6061 (1983).

${ }^{2}$ P. W. Anderson, Phys. Rev. 24, 41 (1961).

${ }^{3}$ A. Okiji and N. Kawakami, J. Phys. Soc. Jpn. 51, 1145 (1982).

${ }^{4}$ P. B. Wiegmann, Phys. Lett. 80A, 163 (1980); A. Okiji and Kawakami, J. Phys. Soc. Jpn. 51, 3192 (1982); N. Kawakami and A. Okiji, ibid. 51, 2043 (1982); K. G. Wilson, Rev. Mod.
Phys. 47, 773 (1975); K. Yosida and K. Yamada, Prog. Theor. Phys. 46, 244 (1970); 53, 970 (1975); 53, 1286 (1975); B. Horvatić and V. Zlatić, Phys. Rev. B 30, 6717 (1984); 28, 6904 (1983).

${ }^{5}$ The variational parameters are the components of the eigenvectors of the Hamiltonian matrix. See B. N. Parlett, The Symmetric Eigenvalue Problem (Prentice-Hall, Englewood Cliffs, 1980).

${ }^{6}$ C. M. Varma and Y. Yafet, Phys. Rev. B 13, 2950 (1976); O. Gunnarsson and K. Schönhammer, ibid. 31, 4815 (1985). 